\title{
New Insights into X-ray Binaries
}

Jorge Casares

\begin{abstract}
X-ray binaries are excellent laboratories to study collapsed objects. On the one hand, transient X-ray binaries contain the best examples of stellar-mass black holes while persistent X-ray binaries mostly harbour accreting neutron stars. The determination of stellar masses in persistent $\mathrm{X}$-ray binaries is usually hampered by the overwhelming luminosity of the X-ray heated accretion disc. However, the discovery of high-excitation emission lines from the irradiated companion star has opened new routes in the study of compact objects. This paper presents novel techniques which exploits these irradiated lines and summarises the dynamical masses obtained for the two populations of collapsed stars: neutron stars and black holes.
\end{abstract}

\section{Introduction}

$\mathrm{X}$-ray binaries (XRBs hereafter) are interacting binaries where $\mathrm{X}$-rays arise from the accretion of matter onto a neutron star (NS) or a black hole (BH). Accretion processes are found in other astrophysical environments such as cataclysmic variables (i.e. interacting binaries with accreting white dwarfs), $T$ Tauri stars, protoplanetary discs, etc. but the unique property of XRBs is the presence of central compact objects that are the remnants of collapsed, massive stars. Therefore, they provide the best laboratories to study their properties in detail, such as masses, spin or NS equation of state. This paper is not meant to give a thorough review of XRBs but instead it will focus on three selected topics with implications for our knowledge of the mass spectrum of collapsed stars. These are:

1. Evidence for BHs in XRBs: a summary of dynamical masses is presented.

2. The Bowen Project: a new technique to trace the orbit of companion (donor) stars in persistent XRBs.

J. Casares

Instituto de Astrofísica de Canarias, E-38200 La Laguna, Tenerife, Spain, e-mail: jcv@iac.es 
3. Echo Tomography: reprocessed light from the donor star can constrain the binary inclination, the parameter with largest impact in the mass error.

Excellent reviews on other aspects of XRBs can be found in [9] and [24].

\section{Black Holes in X-ray Binaries}

The mass distribution of Black Holes (BHs hereafter) has strong impact in several areas of Astrophysics, in particular SNe models, the evolution of massive stars, chemical enrichment of the galaxy, jet formation etc.. Stellar evolution theories predict $\sim 10^{9}$ BHs remnants in the Galaxy [2] but only BHs in interacting binaries can be easily detected through $\mathrm{X}$-ray radiation triggered by accretion. This is the reason why the history of $\mathrm{BH}$ discoveries has run in parallel with the development of X-ray astronomy.

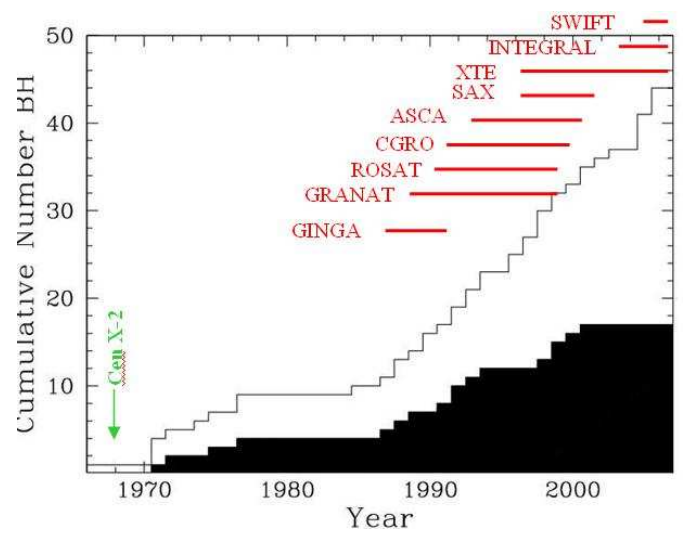

Fig. 1 Cumulative distribution of $\mathrm{BH}$ SXTs discovered during the era of X-ray astronomy. The black histogram indicates dynamical $\mathrm{BHs}$

In particular Soft X-ray transients (SXTs hereafter) provide the best systems to find stellar-mass BHs, since $\sim 75 \%$ of these transients likely harbour a BH. SXTs are a subclass of X-ray binaries with low-mass donor stars (typically of K-M spectral types) which exhibit episodic outbursts due to mass transfer instabilities in the accretion disc [23]. During outburst, the X-ray luminosity increases by factors up to $10^{6}-10^{7}$ and, therefore, they are easily spotted by X-ray satellites. Unfortunately, the companion star is overwhelmed by the X-ray heated disc at all wavelengths, precluding its detection. However, after a few months of activity, X-rays switch off, the reprocessed light drops off several magnitudes into quiescence and the companion starts to dominate the optical flux. This provides a special opportunity for spectroscopic detection, perform dynamical studies and derive stellar masses. Figure 1 shows the cumulative histogram of BH SXTs discovered since 1966, when Cen X-2 was first detected during a rocket flight. A linear increase at a rate of $\sim 1.7$ 
$\mathrm{yr}^{-1}$ is apparent since the late 80 's, when a new fleet of X-ray satellites with higher sensitivity and All-Sky-Monitor capabilities became operative.

The best evidence for the presence of BHs is dynamical, i.e. a compact object whose mass exceeds the maximum allowed for stable neutron stars or $\sim 3 \mathrm{M}_{\odot}$ [36]. And this is relatively easy to prove through the observed radial velocity curve of the companion star during quiescence. The orbital period $P_{\text {orb }}$ and velocity semiamplitude $K_{\mathrm{C}}$ combine in the standard mass function equation $f(M)$

$$
f(M)=\frac{P_{\mathrm{orb}} K_{\mathrm{C}}^{3}}{2 \pi G}=\frac{M_{\mathrm{X}} \sin ^{3} i}{(1+q)^{2}},
$$

which relates the mass of the compact object $M_{\mathrm{X}}$ and the companion star $M_{\mathrm{C}}$ through the orbital inclination $i$ and mass ratio $q=\frac{M_{\mathrm{C}}}{M_{\mathrm{X}}}$. It is easy to show that $f(M)$ yields a secure lower limit to the $\mathrm{BH}$ mass $M_{\mathrm{X}}$. This experiment typically requires resolving powers $\geq 1500$ and can be performed with targets brighter than $R \sim 23$ using current instruments on 10m-class telescopes.

But getting actual $\mathrm{BH}$ masses rather than lower limits is not so straightforward. The reason being that, by their very nature, $\mathrm{BHs}$ do not burst nor pulse and hence one cannot trace their orbital motion. We are facing a single-line spectroscopic binary where the extra observables ( $q$ and $i$ ) must be extracted from the optical star. And this can be accomplished with two further experiments:

- Resolving the rotational broadening of the donor's photospheric lines. Because the donor star is filling the Roche lobe and synchonized, the rotational broadening $V \sin i$ scales with $K_{\mathrm{C}}$ as a function of $q$ [42]. Therefore, by measuring $V \sin i$ one can determine $q$ directly.

- Fitting synthetic models to the ellipsoidal modulation. The changing visibility of the tidally distorted companion star generates a double-humped light curve (the so-called ellipsoidal modulation) whose amplitude is a strong function of $i$ and $q$. For extreme mass ratios $q<0.2$, typical of BH binaries, the shape of the light curve is weakly sensitive to $q$ and hence $i$ can be easily determined [38].

By combining the mass function with constraints on $q$ and $i$ one gets a full dynamical solution and hence the BH mass with minimum assumptions. Further details on this prescription and possible systematics involved can be found in several reviews such as [3].

BHs have also been found in a handful of High Mass X-ray Binaries (HMXBs hereafter), i.e. XRBs with early-type massive donor stars. However, here we find several limitations which complicate the analysis. A key factor is $M_{\mathrm{C}}$, which for a HMXB is a large number and has a wide range of uncertainty. The optical star is likely to be undermassive for its spectral type as a result of mass transfer and binary evolution [35]. Furthermore, mass transfer is usually produced through winds rather than Roche lobe overflow and this has a two-fold effect. On one side, wind emission can contaminate the radial velocities of the donor star. On the other, since the optical star does not fill its Roche lobe, $q$ and $i$ values derived through $V \sin i$ and ellipsoidal fits may be overestimated. These caveats can only be side-lined in eclipsing binaries 
such as X-7 in M33, which so far is the only case where this has been possible. In addition to the eclipse duration, the distance provides an extra restriction which lead to tight constraints in the space parameter. In particular, the radius of the donor, the Roche lobe filling factor and the inclination are accurately determined and yield a very precisse $\mathrm{BH}$ mass [33].

Table 1 Dynamical BHs

\begin{tabular}{|c|c|c|c|c|c|}
\hline System & $\begin{array}{c}P_{\text {orb }} \\
\text { [days] }\end{array}$ & $\begin{array}{l}f(M) \\
{\left[M_{\odot}\right]}\end{array}$ & $\begin{array}{c}\text { Donor } \\
\text { Spect. Type }\end{array}$ & Classification & $\begin{array}{l}M_{\mathrm{x}}^{\dagger} \\
{\left[M_{\odot}\right]}\end{array}$ \\
\hline GRS $1915+105^{a}$ & 33.5 & $9.5 \pm 3.0$ & K/M III & LMXB/Transient & $14 \pm 4$ \\
\hline V404 Cyg & 6.471 & $6.09 \pm 0.04$ & K0 IV & & $12 \pm 2$ \\
\hline Cyg X-1 & 5.600 & $0.244 \pm 0.005$ & $09.7 \mathrm{Iab}$ & HMXB/Persistent & $10 \pm 3$ \\
\hline $\mathrm{LMC} X-1^{b}$ & 3.909 & $0.143 \pm 0.007$ & 07 III & & $10.3 \pm 1.3$ \\
\hline M33 X-7 & 3.453 & $0.46 \pm 0.08$ & 07-8 III & 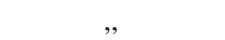 & $15.7 \pm 1.5$ \\
\hline XTE J1819-254 & 2.816 & $3.13 \pm 0.13$ & B9 III & IMXB/Transient & $7.1 \pm 0.3$ \\
\hline GRO J1655-40 & 2.620 & $2.73 \pm 0.09$ & F3/5 IV & & $6.3 \pm 0.3$ \\
\hline $\mathrm{BW} \mathrm{Cir}^{c}$ & 2.545 & $5.73 \pm 0.29$ & G5 IV & LMXB/Transient & $>7.0$ \\
\hline GX $339-4^{d}$ & 1.754 & $5.8 \pm 0.5$ & - &, & $>6.0$ \\
\hline LMC X-3 & 1.704 & $2.3 \pm 0.3$ & B3 V & HMXB/Persistent & $7.6 \pm 1.3$ \\
\hline XTE J1550-564 & 1.542 & $6.86 \pm 0.71$ & G8/K8 IV & LMXB/Transient & $9.6 \pm 1.2$ \\
\hline 4U 1543-475 & 1.125 & $0.25 \pm 0.01$ & A2 V & IMXB/Transient & $9.4 \pm 1.0$ \\
\hline H1705-250 & 0.520 & $4.86 \pm 0.13$ & $\mathrm{~K} 3 / 7 \mathrm{~V}$ & LMXB/Transient & $6 \pm 2$ \\
\hline GS 1124-684 & 0.433 & $3.01 \pm 0.15$ & $\mathrm{~K} 3 / 5 \mathrm{~V}$ &, & $7.0 \pm 0.6$ \\
\hline XTE J1859+226 ${ }^{e}$ & 0.382 & $7.4 \pm 1.1$ & - & $"$ & \\
\hline GS2000+250 & 0.345 & $5.01 \pm 0.12$ & $\mathrm{~K} 3 / 7 \mathrm{~V}$ & & $7.5 \pm 0.3$ \\
\hline A0620-003 & 0.325 & $2.72 \pm 0.06$ & K4 V & , & $11 \pm 2$ \\
\hline XTE J1650-500 & 0.321 & $2.73 \pm 0.56$ & K4 V & , & \\
\hline GRS 1009-45 & 0.283 & $3.17 \pm 0.12$ & K7/M0 V & ( & $5.2 \pm 0.6$ \\
\hline GRO J0422+32 & 0.212 & $1.19 \pm 0.02$ & M2 V & $"$ & $4 \pm 1$ \\
\hline XTE J1118+480 & 0.171 & $6.3 \pm 0.2$ & K5/M0 V & , & $6.8 \pm 0.4$ \\
\hline
\end{tabular}

${ }^{\dagger}$ Masses compiled by [9] and [32].

${ }^{a}$ New photometric period of $30.8 \pm 0.2$ days reported by [30].

${ }^{b}$ Updated after [34.

${ }^{c}$ Updated after [7].

${ }^{d}$ Updated after [27].

${ }^{e}$ Period is uncertain. See [43].

Table 1 presents an updated list of confirmed BHs based on dynamical arguments, with their best mass estimates. We currently have $21 \mathrm{BHs,} \mathrm{with} \mathrm{orbital} \mathrm{pe-}$ riods between 33.5 days and 4.1 hours. The great majority are SXTs (17) while 4 are persistent HMXBs: Cyg X-1 plus the 3 extragalactic binaries LMC X-1, LMC X-3 and M33 X-7. The case of GX 339-4 deserves special mention because it is the only SXT where the presence of a BH was proven during the outburst phase. This was possible thanks to the detection of fluorescent lines arising from the irradiated companion (see Sect. 3). GRS 1915+105 is also noteworthy, not only because of its long orbital period and large mass function but also because IR spectroscopy was essential to overcome the $>25$ magnitudes of optical extinction and reveal the radial 
velocity curve of the companion star [20]. However, it should be noted that recent photometry reports a slightly shorter orbital period and evidence for irradiated light curves [30]. The combination of these two effects will likely decrease the mass function and BH mass. XTE J1859+226 also needs revisiting because its orbital period is uncertain [43]. In summary we have $16 \mathrm{BH}$ masses ranging between 4 and $16 \mathrm{M}_{\odot}$ with $\sim 5-30 \%$ errors. These can be compared with theoretical distributions of stellar remnants such as [19]. The model includes binary interaction under Case $\mathrm{C}$ mass transfer (i.e. Common Envelope evolution after core helium ignition), wind mass-loss in the Wolf-Rayet phase and SN Ib explosion. The computation predicts a continuum distribution of remnants with a mass cut at $12 \mathrm{M}_{\odot}$ which is difficult to reconcile with some of the observed masses. However, the model entails many theoretical uncertainties which dominate the final mass spectrum such as the Common Envelope efficiency, the wind mass-loss rate or the progenitor's mass cut. Clearly more SXT discoveries and lower uncertainties in BH masses are required before these issues can be addressed and the form of the distribution is used to constrain $\mathrm{BH}$ formation models and XRB evolution.

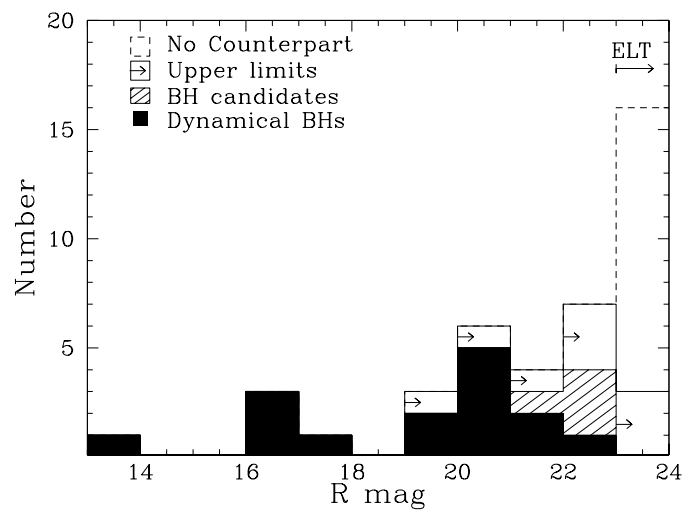

Fig. 2 Magnitude distribution of BH SXTs in quiescence. The black histogram indicates dynamical $\mathrm{BHs}$ while the rest are $\mathrm{BH}$ candidates. Targets without optical counterpart are likely fainter than $R>23$.

In addition to dynamical BHs, there are 27 other SXTs with similar X-ray spectral and timing properties during outburs 1 . Unfortunately, these $\mathrm{BH}$ candidates become too faint in quiescence for dynamical studies or even lack accurate astrometry. This is illustrated in Fig. 2 which shows the magnitude distribution of the 44 currently known BH transients. Dynamical studies are only possible with the current largest telescopes for sources brighter than $R \leq 23$. Not shown in the figure is the heavily reddened GRO $1915+105$ which was studied in the NIR. The figure depicts the bright tail of a dormant population of galactic BH SXTs which several works have estimated in a few thousand systems ([37] and included references). Im-

${ }^{1}$ This number has been updated after [24] with new detections reported in several Astronomical Telegrams. 
proving the statistics of dynamical BHs requires not only a new generation of ELT telescopes to tackle fainter targets but also new strategies aimed at unveiling new "hibernant" SXTs before they go into outburst. Quiescent BH SXts typically have EW $\left(\mathrm{H}_{\alpha}\right) \sim 20-50 \AA$ and hence they should show up in deep $\mathrm{H}_{\alpha}$ surveys such as IPHAS [17]. However, clever diagnostics need to be defined to clear out other populations of $\mathrm{H}_{\alpha}$ emitters such as cataclysmic variables or T Tauris (see Corral-Santana et al., these proceedings).

\section{The Bowen Project}

Aside from transient XRBs, there are $\sim 150$ persistent XRBs in the Galaxy, the great majority hosting neutron stars (NS hereafter) accreting at the Eddington limit. They are considered the progenitors of Binary Millisecond Pulsars (BMPs hereafter) because is the sustained accretion during their long active lives that spins the NS up to millisecond periods. The discovery of millisecond pulses in 8 transient XRBs and coherent oscillations during X-ray bursts in 13 persistent XRBs gave strong support to this "recycle" pulsar scenario. And burst oscillations were detected in addition to persistent pulses in the transient XRBs SAX J1808-3658 [10] and XTE J1814338 [40] with identical frequencies. This confirmed that burst oscillations are indeed modulated with the spin of the NS. The interest of these discoveries stands in the fact that one can use the orbital Doppler shift of pulses/oscillations to trace the NS orbit and obtain the X-ray mass function.

Optical emission in persistent XRBs is triggered by reprocessing of the intense $\mathrm{X}$-ray radiation in different binary sites, mainly the accretion disc. The companion star is $\sim 1000$ times fainter than the irradiated disc at optical-IR wavelengths and hence completely undetectable. This has systematically plagued attempts to determine system parameters and, in most cases, only the orbital period is known. Fortunately, there are methods which can exploit the effects of irradiation and X-ray variability. New prospects were opened by the discovery of sharp high excitation emission lines arising from the irradiated face of the companion star in Sco X-1 [39]. The most prominent are found in the core of the Bowen feature, a blend of CIII/NIII lines which are mainly powered by fluorescence. These lines trace the motion of the companion star and provided the first dynamical information on this protypical LMXB (see Fig. 3). Since then, sharp Bowen lines from companion stars have been discovered in 7 other persistent LMXBs and 4 transients during outburst: Aql X-1, GX 339-4 and the BMPs XTE J1814-338 and SAX J1808.4-3658. These transient studies beautifully demonstrate the power of this technique in systems which otherwise cannot be studied in quiescence because either they are too faint (case of GX 339-4 and the BMPs) or are contaminated by a bright interloper (Aql X-1). In particular, the case of GX 339-4 is remarkable because the Bowen study provides the first solid evidence for the presence of a $\mathrm{BH}$ in this classic transient.

The radial velocity curves of the Bowen lines are biased because they arise from the irradiated face of the star instead of its center of mass. Therefore, a K-correction 

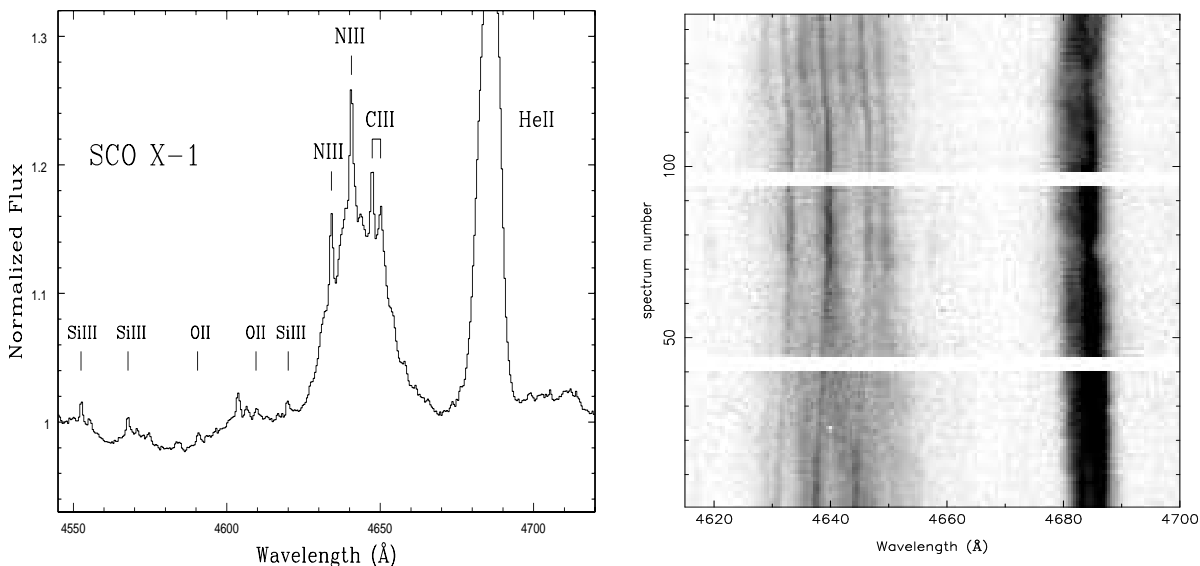

Fig. 3 Detecting companion stars in persistent XRBs. Left: the main high excitation emission lines due to irradiation of the donor star in Sco X-1. Adapted from [39]. Right: Trail spectrum showing the radial velocity motion of the Bowen CIII/NIII lines as a function of time. After [39].

needs to be applied in order to obtain the true velocity semi-amplitude $K_{\mathrm{C}}$ from the observed velocity $K_{\mathrm{em}}$. The $K$-correction parametrizes the displacement of the center of light with respect to the donor's center of mass through the mass ratio and disc flaring angle $\alpha$. The latter dictates the size of the disc shadow projected over the irradiated donor [25]. Extra information on $q$ and $\alpha$ is thus required to get the real $K_{\mathrm{C}}$. Furthermore, useful limits to the NS mass can be set if the binary inclination is well constrained through eclipses.

Table 2] summarises the NS masses obtained through the Bowen technique during several campaigns at the WHT, AAT and VLT. The list of persistent systems is almost a complete sample of Galactic LMXBs brigther than $\mathrm{B} \simeq 19$. In the cases of Aql X-1 and X1822-371 the evidence of NS more massive than canonical is very persuasive. The latter is a particularly favourable binary because it is eclipsing and the NS is a pulsar. Then its radial velocity curve is known through the study of orbital pulse delays. Good constraints on the NS velocity are also available for V801 Ara through the detection of pulse oscillations during a superburst [6]. Tight limits to the inclination and mass ratio are also available for the eclipsing EXO 0748676 [29] and the dipper GR Mus [1]. In the remaining cases the NS mass is not well constrained due to large uncertainties in the inclination and/or mass ratio. However, it is important to stress that these are the first dynamical constraints in persistent LMXBs since their discovery, 40 years ago. Other techniques (such as the Echo Tomography) need to be exploited to further refine these limits and derive more accurate NS masses. Previous reviews presenting results of the Bowen project can be found in [5] and [14]. 
Table 2 NS masses obtained using the Bowen Technique

\begin{tabular}{|c|c|c|c|c|c|c|}
\hline System & $\begin{array}{l}P_{\text {orb }} \\
{[\mathrm{hr}]}\end{array}$ & Mag & Type & $\begin{array}{l}K_{\mathrm{em}} \\
{[\mathrm{km} / \mathrm{s}]}\end{array}$ & $\begin{array}{l}M_{\mathrm{NS}}^{\dagger} \\
{\left[M_{\odot}\right]}\end{array}$ & Reference \\
\hline Sco X-1 & 18.9 & $\mathrm{~B}=12.2$ & Persistent & $87 \pm 1$ & $>0.2$ & 39 \\
\hline LMC X-2 & 8.1 & $\mathrm{~B}=18$ & , & $351 \pm 28$ & $>1.2$ & 13] \\
\hline $\mathrm{X} 1822-371$ & 5.6 & $\mathrm{~B}=15.8$ & , & $300 \pm 15$ & $1.6-2.3$ & [4] \\
\hline V926 Sco (X1735-444) & 4.7 & $\mathrm{~B}=17.9$ & , & $226 \pm 22$ & $>0.5$ & [6] \\
\hline GX9+9 (X1728-16) & 4.2 & $\mathrm{~B}=16.8$ & , & $230 \pm 35$ & $>0.3$ & 12 \\
\hline GR Mus & 3.9 & $\mathrm{~B}=19.1$ & , & $245 \pm 30$ & $1.2-2.6$ & [1] \\
\hline V801 Ara (X1636-536) & 3.8 & $\mathrm{~B}=18.2$ & , & $277 \pm 22$ & $>0.8$ & [6] \\
\hline EXO 0748-676 & 3.8 & $B=16.9$ & , & $310 \pm 10$ & $1.1-2.6$ & 29 \\
\hline Aql X-1 & 19 & $\mathrm{~V} \sim 22$ & Transient & $247 \pm 8$ & $>1.6$ & [11 \\
\hline GX 339-4 & 42.1 & $\mathrm{~V}>21$ & , & $317 \pm 10$ & $>6.0$ & 21 27 \\
\hline XTE J1814-338 ${ }^{a}$ & 4.2 & $\mathrm{~V}=23.3$ & Transient/BMP & $345 \pm 19$ & $>1.0$ & [8] \\
\hline SAX J1808.4-3658 & 2.0 & $\mathrm{~V}=21.8$ & , = - n & $248 \pm 20$ & $>0.3$ & [15 \\
\hline
\end{tabular}

${ }^{\dagger}$ After K-correction and constraints to the inclination, mass ratio or NS velocity (when available).

${ }^{a}$ Preliminary results.

\section{Echo Tomography}

Echo Tomography uses time delays between X-ray and UV/optical variability as a function of orbital phase to map the reprocessing sites in a binary [31]. The optical variability can be modelled by the convolution of the X-ray light curve with a transfer function which depends on the binary geometry. The transfer function encodes information on the most fundamental parameters such as the binary inclination, star separation and mass ratio. And in particular, the component associated with the companion star is most sensitive to these parameters so detecting echoed emission from the donor offers the best opportunity to constrain them. There has been several attempts at detecting correlated optical and X-ray variability using white light or broad band filters (e.g. [41], [22]). These works have detected delays which are mostly consistent with reprocessing in the outer disc implying that the disc is the dominant source of continuum reprocessed light.

Exploiting emission-line reprocessing rather than broad-band photometry has two potential benefits: a) it amplifies the response of the donor's contribution by suppressing most of the background continuum light (dominated by the disc); b) since the emission line reprocessing time is instantaneous, the response is sharper (i.e. only smeared by geometry). Through the Bowen project we know that high energy radiation is very efficiently reprocessed by the donor's atmospheres into Bowen fluorescence lines. Therefore, we decided to search for optical echoes of X-ray variability using ULTRACAM [16] equipped with a special set of narrow band filters, centered at the Bowen blend and a red continuum. The latter is essential to subtract the continuum light and hence amplify the reprocessed signal from the companion. During an RXTE/WHT campaign on Sco X-1 correlated variability was detected at 

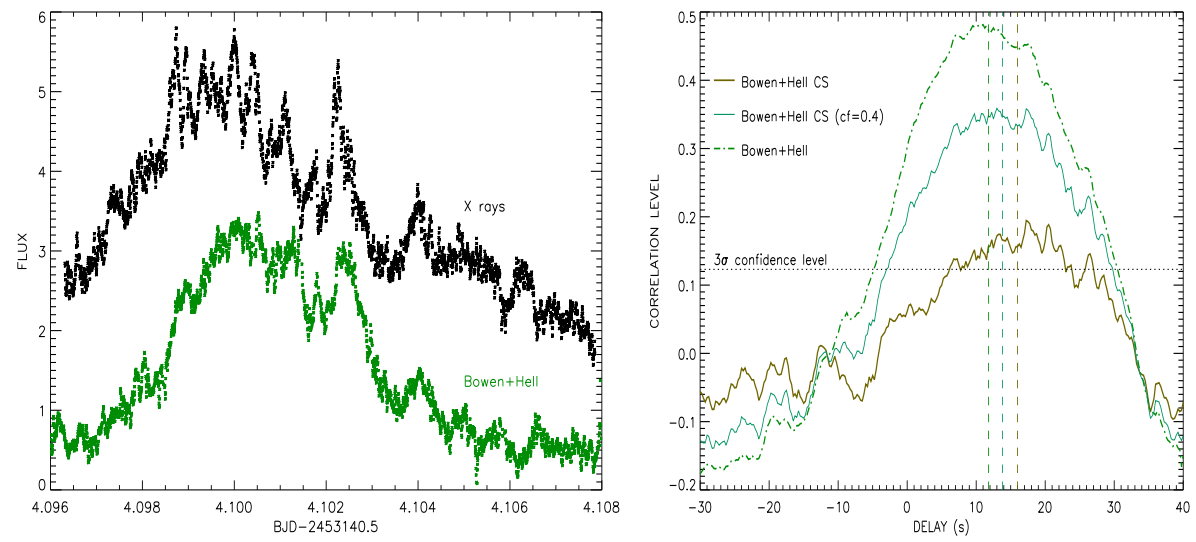

Fig. 4 Echo Tomography experiment in Sco X-1. Left: large amplitud X-ray variability and correlated optical light curve observed at orbital phase 0.5 . Sco X-1 happened to be in the flaring branch state. Right: Cross-correlation functions between X-rays and optical light curves observed in the continuum (top), Bowen+HeII window (middle) and Bowen+HeII after continnum subtraction (bottom). After [26].

phase $\simeq 0.5$ i.e. superior conjunction of the companion star, when the heated face presents its maximum visibility [26]. Time delays of 14-16s are measured after the continuum light is subtracted from the Bowen light curves (see Fig. 4). These delays are consistent with the light traveltime between the NS and the companion star and hence provide the first evidence of reprocessing in the companion of Sco X-1. However, one needs to detect several optical echoes as a function of orbital phase in order to constrain $i$ and $q$ and derive masses.

In a second campaign we observed the burster X1636-536 simultaneously with RXTE and VLT+ULTRACAM. Three X-ray bursts and their corresponding optical echoes were recorded at orbital phases $0.55,0.20$ and 0.83 and these are shown in the left panel of Fig. 5. The optical bursts clearly lag X-ray burst and are also smeared, indicating an extended reprocessing site. Delay times are in the range 2-3 secs showing little evidence for orbital variability. However, these delays drift when several amounts of continuum light (parametrised by the factor $c f$ ) are subtracted from the Bowen+HeII light. And for $c f \simeq 0.8-0.95$ the 3 delays become consistent with reprocessing in the companion for $\mathrm{M}_{\mathrm{NS}}=1.4 \mathrm{M}_{\odot}, q=0.3, \alpha=12^{\circ}$ and $i=36-60^{\circ}$, as derived through radial velocities of the Bowen lines [6]. This is illustrated in the right panel of Fig. 5. Note that, in particular, delays observed at phase $\sim 0.5$ are especially sensitive to the inclination angle. The main difficulty which hinders us from constraining the inclination is the unknown amount of continuum substraction. In principle, there must be an optimum $c f$ factor which results in a perfect subtraction. However, this is not easy to find because the continuum 

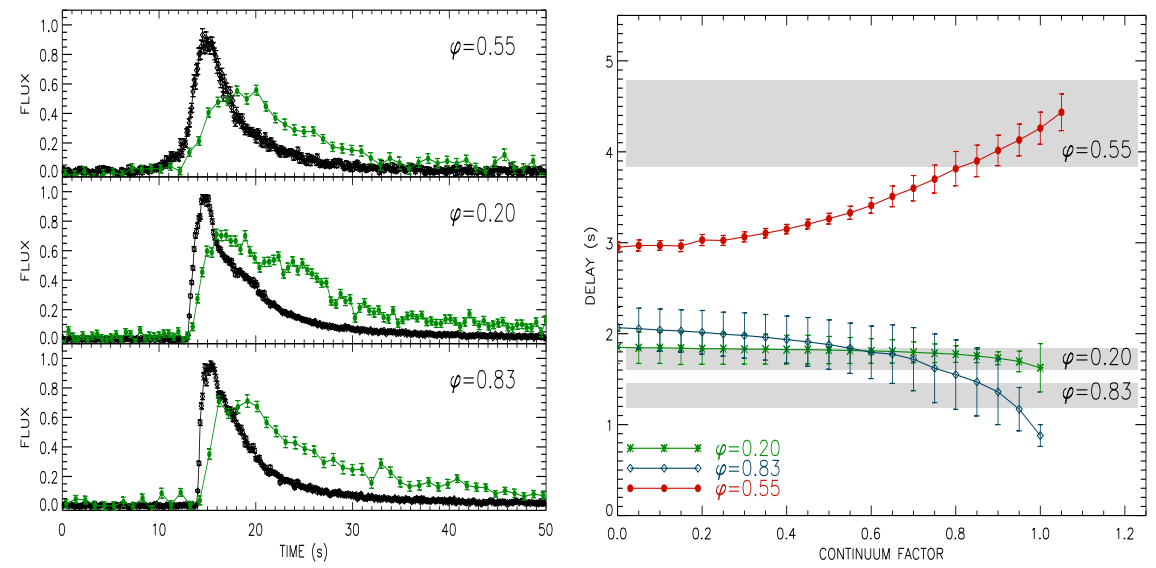

Fig. 5 Echo Tomography of X1636-536. Left: the three X-ray bursts detected and their optical (Bowen+HeII) counterparts. Right: delay times between the X-ray and Bowen+HeII burst light curves as a function of continuum subtraction factor. Shaded regions correspond to delays expected for reprocessing in the companion at each orbital phase. They are computed for $\mathrm{M}_{\mathrm{NS}}=1.4 \mathrm{M}_{\odot}$, $q=0.3, i=36-60^{\circ}$ and $\alpha=12^{\circ}$. After [28].

filter is placed $\sim 1500 \AA$ away from the Bowen lines due to the optical layout of ULTRACAM. New high-speed spectrophotometry devices such as ULTRASPEC will provide pure emission line light curves for echo mapping experiments. These are likely to yield accurate inclinations and, when combined with dynamical information from the Bowen lines and X-ray mass functions, the first accurate NS masses in persistent XRBs.

\section{Conclusions}

In the past 20 years the field of X-ray binaries has experienced significant progress with the discovery of 17 new BHs and 8 transient BMPs in LMXBs. Dynamical masses are available for 16 BHs but better statistics and improved errors are required before using the observed distribution to constrain XRB evolution and supernova models. Exploiting deep $\mathrm{H} \alpha$ surveys of the Galactic plane, such as IPHAS, may unveil a significant fraction of a large expected population of quiescent XRBs. 
The discovery of fluorescence emission from the companion star has opened the door to derive NS masses in persistent and new transient XRBs. This is possible thanks to: i) dynamical information from irradiated donors through high-resolution spectroscopy of the Bowen blend; ii) echo-mapping reprocessing sites through simultaneous Bowen-line/X-ray lightcurves. These techniques, together with results from burst oscillations and transient BMPs, will likely provide the first accurate NS masses in XRBs in the near future and perhaps confirm the existence of massive NS. Thanks to these new techniques, which have proven their worth, the future is bright as new instruments and telescopes will allow to push ahead our sample of BHs and NS masses. High-speed and high-resolution instruments, such as OSIRIS at GTC, RSS at SALT and ULTRASPEC, will play a crucial role in this goal.

Acknowledgements I would like to acknowledge helpful comments from my colleagues D. Steeghs, R. Cornelisse and T. Muñoz-Darias. I'm also grateful for support from the Spanish MEC grant AYA2007-66887. Partially funded by the Spanish MEC under the Consolider-Ingenio 2010 Program grant CSD2006-00070: First Science with the GTC.

\section{References}

1. Barnes A. D., Casares J., Cornelisse R., Charles P. A., Steeghs D., Hynes R.I., O’Brien K, 2007, MNRAS, 380, 1182.

2. Brown G. E., Bethe H. A. 1994, ApJ, 423, 659

3. Casares J. 2001, in: F. C. Lazaro \& M. J. Arevalo (eds.), Binary Stars: Selected Topics on Observations and Physical Processes, LNP 563, p. 277

4. Casares J., Steeghs D., Hynes R.I., Charles P.A, O’Brien K. 2003, ApJ, 590, 1041.

5. Casares J., Steeghs D., Hynes R.I., Charles P.A., Cornelisse R., O’Brien K. 2004, Rev Mex $A A, 20,21$.

6. Casares J., Cornelisse R., Steeghs D., Charles P.A, Hynes R.I., O’Brien K., Strohmayer T. E. 2006, MNRAS, 375, 1463

7. Casares J. et al. 2009, ApJ Supp. Series, in press.

8. Casares J. et al., 2009, in preparation

9. Charles P. A., Coe M. J. 2006, in: W. H. G. Lewin \& M. van der Klis (eds.), Compact Stellar X-ray Sources, Cambridge Astrophysics Series No. 39 (Cambridge: Cambridge University Press), p. 215

10. D. Chakrabarty, E.H. Morgan, M.P. Muno, D.K. Galloway, R. Wijnands, M. van der Klis \& C.B. Markwardt 2003, Nature, 424, 42.

11. Cornelisse R., Casares J., Steeghs D., Barnes A. D., Hynes R.I., O’Brien K, 2007, MNRAS, 375,1463

12. Cornelisse R., Steeghs D., Casares J., Charles P. A., Barnes A. D., Hynes R.I., O’Brien K, 2007, MNRAS, 380, 1219.

13. Cornelisse R., Steeghs D., Casares J., Charles P. A., Shih I.C., Hynes R.I., O’Brien K, 2007, MNRAS, 381, 194.

14. Cornelisse R., Casares J., Muñoz-Darias T., Steeghs D., Charles P.A., Hynes R.I., O'Brien K., Barnes A., 2008, in A POPULATION EXPLOSION: The Nature \& Evolution of X-ray Binaries in Diverse Environments. AIP Conf. Proc., Volume 1010, p. 148. 
15. Cornelisse R. et al., 2009, A\&A, in press.

16. Dhillon V.S. et al., 2007, MNRAS, 378, 825.

17. Drew J.E. et al., 2005, MNRAS, 362, 753.

18. Friedman J. L., Ipser J.R. 1987, ApJ, 314, 594

19. Fryer, C.L. \& Kalogera, V. 2001, ApJ, 554, 548.

20. Greiner J., Cuby J. G., McCaughrean M. J. 2001, Nature, 414, 522

21. Hynes R. I., Steeghs D., Casares J., Charles P. A., O’Brien K. 2003, ApJ, 583, L95

22. Hynes R.I., Correlated X-ray and Optical Variability in X-ray Binaries, 2005, ASP Conf. Ser., Vol.330, Ed. J.-M. Hameury and J.-P. Lasota. San Francisco: Astronomical Society of the Pacific, p.237-250.

23. King A. R. 1999, Phys. Rev., 311, 337

24. McClintock J. E., Remillard R. A. 2006, in: W. H. G. Lewin \& M. van der Klis (eds.), Compact Stellar X-ray Sources, Cambridge Astrophys. Ser. No. 39 (Cambridge: Cambridge University Press), p. 157

25. Muñoz-Darias T., Casares J., Martínez-Pais I. G. 2005, ApJ, 635, 502

26. Muñoz-Darias T., Martínez-Pais I. G., Casares J., Dhillon V. S., Marsh T.R., Cornelisse R., Steeghs D., Charles P. A. 2007, MNRAS, 379, 1673

27. Muñoz-Darias T., Casares J., Martínez-Pais I. G. 2008, MNRAS, 385, 2205

28. Muñoz-Darias T. et al. 2008, in HIGH TIME RESOLUTION ASTROPHYSICS: The Universe at Sub-Second Timescales. AIP Conf. Proc., Volume 984, p. 15.

29. Munõz-Darias T. et al. 2009, in preparation

30. Neil E. T., Bailyn C. D., Cobb B. E. 2006, ApJ, in press (astro-ph/0610480)

31. O'Brien K., Horne K., Hynes R.I., Chen W., Haswell C.A., Still M.D., 2002, MNRAS, 334, 426-434.

32. Orosz, J.A. 2003, in: K. A. van der Hucht, A. Herrero \& C. Esteban (eds.) A Massive Star Odyssey, from Main Sequence to Supernova, Proc. IAU Symp. No. 212 (San Francisco: Astronomical Society of the Pacific) p. 365

33. Orosz J.A. et al. 2007, Nature, 449, 872.

34. Orosz J.A. et al. 2008, arXiv:0810.3447.

35. Rappaport S. A., Joss P. C.. 1983, in: W. H. G. Lewin \& E. P. J.. van den Heuve (eds.) Accretion-driven X-ray Sources, (Cambridge: Cambridge University Press), p. 33

36. Rhoades C. E., Ruffini R. 1974, Phys. Rev. Lett., 32, 324

37. Romani R. W. 1998, A\&A, 333, 583

38. Shahbaz T., Naylor T., Charles P.A. 1994, MNRAS, 268, 756.

39. Steeghs D., Casares J. 2002, ApJ, 568, 273

40. T.E. Strohmayer, C.B. Markwardt, J.H. Swank \& J.J.M.in't Zand 2003, ApJ, 596, 67.

41. van Paradijs J., van der Klis M., van Amerongen S. et al., 1990, A\&A, 234, 181-187.

42. Wade R.A.., Horne K., 1988, ApJ, 324, 411.

43. Zurita C. et al. 2002, MNRAS, 334, 999 